\title{
Editorial
}

\section{Management of anticoagulants during pregnancy}

Although pregnancy induces a state of hypercoagulability, the thromoboembolic risks during a normal pregnancy are minor. This is not the case when the pregnant women has a native valvulopathy in atrial fibrillation, a mechanical prosthesis or a coagulation anomaly. The respective indications for heparin and oral anticoagulants reflect their respective advantages and disadvantages for the mother and child.

Historically, recommendations for anticoagulants during pregnancy have evolved with each new publication of results concerning their use.

Very early, coumarin induced embryopathies, ${ }^{1}$ especially during the first trimester, led to restricted use of oral anticoagulants in favour of heparin. However, it rapidly became apparent that heparin also had disadvantages, especially in women with prostheses. Heparin in these patients facilitated the evolution of the pregnancy by limiting fetal accidents but increased maternal haemorrhagic and thromboembolic risks.

The ease of use and safety provided by low molecular weight heparins (LMWH) led to their widespread use, other than in pregnancy, to treat and prevent arterial and venous thromboembolic events. At present, their use during pregnancy is hampered by the lack of large randomised prospective studies, which are difficult to conduct in light of the ethical constraints which understandably apply.

\section{Current status of anticoagulation in pregnancy}

The risks associated with oral anticoagulants occur primarily during the second part of the first trimester (embryopathies) and the peripartum period (maternal and fetal haemorrhages). ${ }^{2}$ Outside these critical periods, oral anticoagulants, compared to heparin, better stabilise the intensity of anticoagulation and thereby limit maternal haemorrhagic and thromboembolic risks. Thus, a general consensus has been reached for their use during the second and most of the third trimester. ${ }^{34}$

The differences of opinion bear on the therapeutic approach to be taken at the start and end of the pregnancy.

Heparin significantly increases thromboembolic events, ${ }^{56}$ particularly during the critical moments of changing from heparin to oral anticoagulant, to such an extent that some authors recommend the exclusive use of oral anticoagulants throughout the first trimester. ${ }^{7}$ This approach is also based on the lower frequency of birth defects in the recent publications with, in contrast, a higher rate of spontaneous abortions, among which the presence of malformed fetuses cannot be excluded.

European and US recommendations leave much room for the parents' opinion in the choice of anticoagulant treatment during this critical period. ${ }^{34}$ Indeed, the alternatives are clear: heparin protects the fetus and aggravates maternal risk, oral anticoagulants protect the mother and aggravate fetal risk. ${ }^{8}$ Presented in this manner, it is hard to envisage that many couples will favour the birth of a normal child to the detriment of the mother's functional and even vital prognosis.

Thus, the doctor must intervene in the discussion and advance possibilities that guide the definitive choice, aided by recent publications showing that the risk of embryopathy is very low when the oral anticoagulant dose required to obtain an effective international normalised ratio (INR) is low and it rises only when the daily dose exceeds $5 \mathrm{mg} .{ }^{9}$ When the dose is lower, it is possible to use oral anticoagulants with a very low fetal risk, including during the entire first trimester.

In general, low doses of warfarin may be sufficient when the target INR is between 2 and 3, as in third generation aortic prostheses in patients in sinus rhythm. ${ }^{10}$ Mitral prostheses in patients in atrial fibrillation or sinus rhythm require an INR between 3 and 4.5. In these cases, the anticoagulant dose is usually higher, aggravating the risk of embryopathy; thus the choice of unfractionated heparin (at effective doses in three injections a day with activated partial thromboplastin time at 2-3 times the control) for the end of the first trimester seems logical.

\section{LMWH in patients with prosthetic valves}

This debate will surely be reopened once the efficacy and safety of LMWH has been proven in patients with prosthetic valves. A non-randomised short term study demonstrated the safety and efficacy of LMWH in patients with valve prostheses. ${ }^{11}$ In addition, LMWH successfully prevented or treated venous thromboembolic events in large series of pregnant women. ${ }^{12-14}$ In contrast, the number of reported cases of pregnant women with prostheses and LMWH can be counted on the fingers of one hand. ${ }^{15}{ }^{16}$ One thromboembolic event in such a case, recently reported, ${ }^{17}$ occurred after prolonged and insufficient treatment. Such is the situation that the most recent US guidelines, ${ }^{4}$ despite listing the numerous potential advantages of LMWH, do not yet recommend their use. Thus, for the time being, their administration should be limited to patients with contraindications to unfractionated heparin at therapeutic doses (two subcutaneous injections a day, effective anti-Xa concentration). The addition of low dose aspirin (75$100 \mathrm{mg}$ ) has been advanced as a type IIa recommendation in the US guidelines.

For the end of the third trimester, the classical strategy is to stop oral anticoagulants at the 37 th week and replace them with intravenous or subcutaneous heparin for the last 15 days. Supporters of exclusive oral anticoagulant treatment propose stopping them before a systematically programmed caesarean section at the 37 th week. ${ }^{7}$ This approach also has its drawbacks: premature interruption of the pregnancy and birth of low weight babies, thereby aggravating the classical fetal growth retardation of children born to oral anticoagulant treated women. It represents a non-obstetrical indication for caesarean section, which itself increases venous thromboembolic risk over that of natural childbirth. Inducing labour combined with natural delivery, in the absence of obstetrical indications for caesarean section, seems to be the most logical approach.

Intravenous heparin is given for 48 hours before labour induction, at which time it is stopped, only to be started again 6-12 hours after delivery, and rapidly switched (third to sixth day) to oral anticoagulants, which are not 
contraindicated for breast feeding because of their low transmission into milk.

In summary, the management of anticoagulants during pregnancy has benefited from recent therapeutic progress and a better understanding of the respective risks of heparin and oral anticoagulants. The greatest therapeutic difficulties remain for women with mechanical prostheses, which are fortunately becoming less common because of the reduction in rheumatic heart disease and advances in mitral valve repair surgery.

Service de Cardiologie,

GUY HANANIA

Hôpital Robert-Ballanger,

93602 Aulnay-sous-bois,

France

1 Hall JG, Pauli RM, Wilson KM. Maternal and fetal sequelae of anticoagulation during pregnancy. Am ₹ Med 1980;68:122-40

2 Sareli P, England MJ, Berk MR, et al. Maternal and fetal sequelae of anticoagulation during pregnancy in patients with mechanical heart valve prostheses. Am $\mathcal{F}$ Cardiol 1989;63:1462-5.

3 Gohlke-Bärwolf C, Acar J, Oakley C, et al. Guidelines for prevention of thromboembolic events in valvular heart disease: study group of the working group on valvular heart disease of the European Society of Cardiology. Eur Heart f 1995; 16:1320-30.

4 Bonow RO, Carabelo B, DeLeon AC, et al. ACC/AHA guidelines for the management of patients with valvular heart disease. $\mathcal{f}$ Am Coll Cardiol 1998;32:1486-588.
5 Hanania G, Thomas D, Michel PL, et al. Pregnancy and prosthetic heart valves: a French cooperative retrospective study of 155 cases. Eur Heart $\mathcal{F}$ valves: a French

6 Sbarouni E, Oakley CM. Outcome of pregnancy in women with valve prostheses. Br Heart f 1994; 71:196-201

7 Cotrufo M, de Luca TSL, Calabro R, et al. Coumarin anticoagulation during pregnancy in patients with mechanical valve prostheses. Eur f Cardiothorac Surg 1991;3:300-5.

8 Sadler L, McCowan L, White H, et al. Pregnancy outcomes and cardiac complications in women with mechanical, bioprosthetic and homograft valves. Br 7 Obstet Gynaecol 2000;107:245-53.

9 Vitale N, De Feo M, De Santo LS, et al. Dose-dependent fetal complications of warfarin in pregnant women with mechanical heart valves. $\mathcal{f} \mathrm{Am}$ Coll Cardiol 1999;33:1637-41.

10 Acar J, Iung B, Boissel JP, et al. AREVA: multicenter randomized comparison of low-dose versus standard-dose anticoagulation in patients with mechanical prosthetic heart valves. Circulation 1996;94:2107-12.

11 Montalescot G, Polle V, Collet JP, et al. Low molecular weight heparin after mechanical heart valve replacement. Circulation 2000;101:1083-6.

12 Conard J, Horellou MH, Samama MM. Management of pregnancy in women with thrombophilia. Haemostasis 1999;29(suppl 1):98-104.

13 Sanson BJ, Lensing AWA, Prins MH, et al. Safety of low-molecular-weight heparin in pregnancy: a systematic review. Thromb Haemost 1999;81:66872 .

14 Verstraete M, Prentice CRM, Samama M, et al. A European view on the North American fifth consensus on antithrombotic therapy. Chest North American

15 Lee LH. Low molecular weight heparin for thromboprophylaxis during pregnancy in 2 patients with mechanical mitral valve replacement. Thromb Haemost 1996:76:628-9.

16 Tenconi PM, Gatti L, Acaia B. Low molecular weight heparin in a pregnant woman with mechanical heart valve prosthesis: a case report. Thromb Haemost 1997;79:733.

17 Lev-Ran O, Kramer A, Gurevitch J, et al. Low-molecular weight heparin for prosthetic heart valves: treatment failure. Ann Thorac Surg 2000;69:264-5.

\section{IMAGES IN CARDIOLOGY}

\section{Infective abdominal aortic aneurysm}

An 81 year old woman with hypertension and diabetes mellitus was admitted for evaluation of severe lumbago and a month long history of high fever of unknown origin. Laboratory investigations showed white blood cell count of $0.234 \times 10^{12} / 1$ and a C-reactive protein concentration of $238 \mathrm{mg} / \mathrm{l}$. Aortography (A) and three dimensional imaging reconstructed from abdominal spiral computed tomography (CT) (B) revealed a $60 \mathrm{~mm}$ pseudo-abdominal

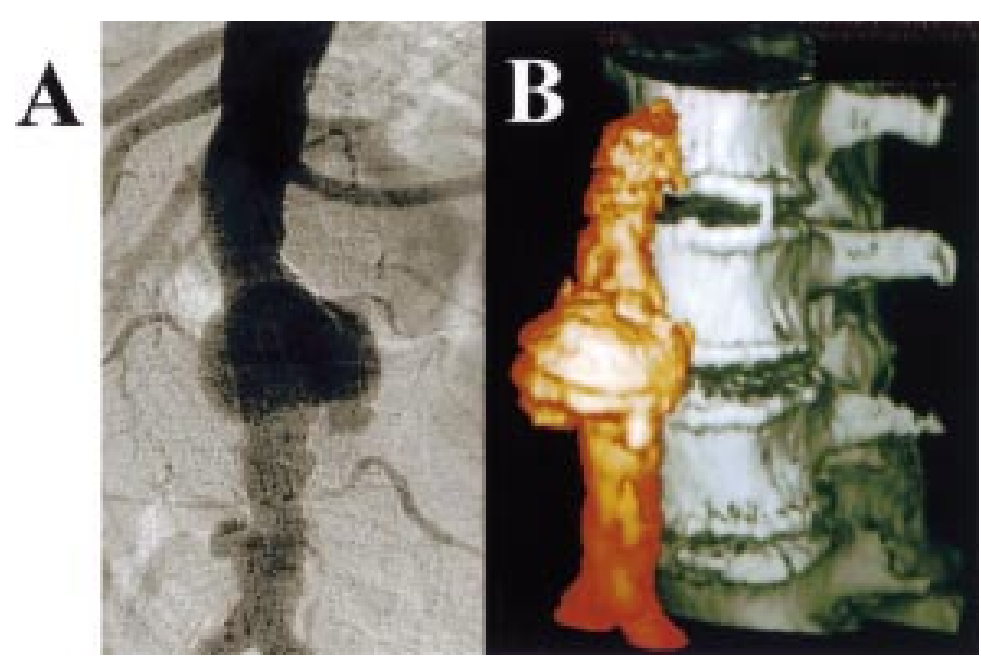

aortic aneurysm with leakage of contrast medium (contrast medium is in colour). Spiral CT images taken from sagittal and coronal angles also demonstrated the aortic aneurysm and leakage of contrast medium in two cavities.

The aneurysm was resected and a bypass graft of right axillar artery to both femoral arteries was used for reconstruction. The aneurysm was $60 \times 70 \mathrm{~mm}$ in diameter with a rupture of $30 \times 20 \mathrm{~mm}$. The aneurysm was divided into two cavities, containing white thrombi caused by a pyemic abscess, and there were adhesions between the upper cavity of the aneurysm and the jejunum with a small penetration into the serosa. Histopathological examinations showed neutrophil infiltrations from the adventitia to the intima of the resected aneurysm, and Escherichia coli was detected by culture of the abscess. The postoperative course was uneventful.

Three dimensional images taken from various angles constitute a non-invasive and useful method to diagnose such serious infectious aortic aneurysms early and to examine the structures in relation to the aneurysm.

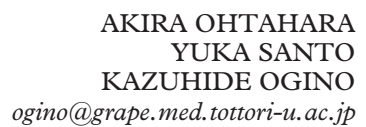

AKIRA OHTAHARA KAZUHIDE OGINO ogino@grape.med.tottori-u.ac.jp 